\title{
An Approximation Bound Analysis for Lasserre's Relaxation in Multivariate Polynomial Optimization
}

\author{
Jiawang Nie
}

Received: 2 June 2013 / Accepted: 5 June 2013 / Published online: 2 July 2013

(C) Operations Research Society of China, Periodicals Agency of Shanghai University, and

Springer-Verlag Berlin Heidelberg 2013

Abstract Suppose $f, g_{1}, \cdots, g_{m}$ are multivariate polynomials in $x \in \mathbb{R}^{n}$ and their degrees are at most $2 d$. Consider the problem:

$$
\text { Minimize } f(x) \text { subject to } g_{1}(x) \geqslant 0, \cdots, g_{m}(x) \geqslant 0 \text {. }
$$

Let $f_{\min }$ (resp., $f_{\max }$ ) be the minimum (resp., maximum) of $f$ on the feasible set $S$, and $f_{\text {sos }}$ be the lower bound of $f_{\text {min }}$ given by Lasserre's relaxation of order $d$. This paper studies its approximation bound. Under a suitable condition on $g_{1}, \cdots, g_{m}$, we prove that

$$
\left(f_{\max }-f_{\mathrm{sos}}\right) \leqslant Q\left(f_{\max }-f_{\min }\right)
$$

with $Q$ a constant depending only on $g_{1}, \cdots, g_{m}$ but not on $f$. In particular, if $S$ is the unit ball, $Q=\mathcal{O}\left(n^{d}\right)$; if $S$ is the hypercube, $Q=\mathcal{O}\left(n^{2 d}\right)$; if $S$ is the boolean set, $Q=\mathcal{O}\left(n^{d}\right)$.

Keywords Approximation bound - Lasserre's relaxation - Polynomials · Semidefinite programming $\cdot$ Sum of squares

Mathematics Subject Classification (2010) 68Q25 · 90C22 · $90 \mathrm{C60}$

The research was partially supported by the National Science Foundation grant DMS-0844775.

J. Nie $(\bowtie)$

Department of Mathematics, University of California San Diego, 9500 Gilman Drive, La Jolla,

CA 92093, USA

e-mail: njw@math.ucsd.edu 


\section{Introduction}

Consider the polynomial optimization problem

$$
\begin{cases}\min _{x \in \mathbb{R}^{n}} & f(x) \\ \text { s.t. } & g_{1}(x) \geqslant 0, \cdots, g_{m}(x) \geqslant 0 .\end{cases}
$$

Here $f, g_{1}, \cdots, g_{m}$ are all multivariate polynomials in $x:=\left(x_{1}, \cdots, x_{n}\right)$. Generally, it is quite difficult to solve (1.1). For instance, when $f(x)$ is a nonconvex quadratic function and every $g_{i}(x)$ is linear, (1.1) becomes a nonconvex quadratic program (QP) which is NP-hard [6]. So problem (1.1) is NP-hard. Lasserre's relaxation [2] is a typical approach for solving (1.1) approximately by using semidefinite programming and sum-of-squares techniques. We refer to [2-5, 7, 8].

When $f, g_{1}, \cdots, g_{m}$ have degrees no greater than $2 d$, Lasserre [2] proposed the following sum-of-squares (SOS) program to find a lower bound for the minimum $f_{\min }$ of (1.1):

$$
\begin{cases}\max & \gamma \\ \text { s.t. } & f-\gamma=\sigma_{0}+\sigma_{1} g_{1}+\cdots+\sigma_{m} g_{m}, \\ & \operatorname{deg}\left(\sigma_{0}\right), \operatorname{deg}\left(\sigma_{1} g_{1}\right), \cdots, \operatorname{deg}\left(\sigma_{m} g_{m}\right) \leqslant 2 d, \\ & \sigma_{0}, \sigma_{1}, \cdots, \sigma_{m} \text { are } \operatorname{SOS} .\end{cases}
$$

Here a polynomial is said to be SOS if it is a sum of squares of polynomials with real coefficients. If a polynomial is SOS, then it must be nonnegative everywhere, but the reverse might not be true. We refer to [10] for a survey on SOS and nonnegative polynomials. While it is quite difficult to check nonnegativity, checking SOS is much easier because it is equivalent to a semidefinite program (SDP) (cf. [7, 8]), which can be solved efficiently. Thus, the SOS program (1.2) would be solved by SDP solvers. The integer $d$ in (1.2) is called the relaxation order.

Here we briefly review the convergence of Lasserre's relaxation (1.2) as $d$ increases for a fixed $f$. For convenience, denote $g=\left(g_{1}, \cdots, g_{m}\right)$ and

$$
S=\left\{x \in \mathbb{R}^{n}: g_{1}(x) \geqslant 0, \cdots, g_{m}(x) \geqslant 0\right\} .
$$

For each $\gamma$ feasible in (1.2), we have

$$
f(x)-\gamma=\sigma_{0}(x)+\sigma_{1}(x) g_{1}(x)+\cdots+\sigma_{m}(x) g_{m}(x) \geqslant 0 \quad \forall x \in S .
$$

Thus, every $\gamma$ feasible in (1.2) satisfies $f(x) \geqslant \gamma$ for all $x \in S$. If we denote by $f_{\text {sos, } d}$ the optimal value of (1.2), then $f_{\text {min }} \geqslant f_{\text {sos, } d}$ for all $d$. As $d$ increases, the lower bound $f_{\mathrm{sos}, d}$ is monotonically increasing. Based on Putinar's Positivstellensatz [9], Lasserre [2] proved $f_{\mathrm{sos}, d} \rightarrow f_{\min }$ as $d \rightarrow \infty$ under a so-called archimedean condition $(A C)$, that is, there exist $M>0$ and SOS polynomials $s_{0}, s_{1}, \cdots, s_{m}$ such that

$$
M-\|x\|_{2}^{2}=s_{0}+s_{1} g_{1}+\cdots+s_{m} g_{m} .
$$

For AC to hold, $S$ must be compact, but the reverse might not be true. However, $\mathrm{AC}$ is not a very strong condition because otherwise we can always add a redundant 
constraint like $M-\|x\|_{2}^{2} \geqslant 0$ if $S$ is compact. Nie and Schweighofer [5] analyzed the convergence rate of (1.2). Under AC, they proved that

$$
0 \leqslant f_{\min }-f_{\text {sos }, d} \leqslant K \cdot(\log d)^{-c} \text { as } d \rightarrow \infty,
$$

where $c>0$ depends only on $g$, and the constant $K=K(f, g)$ depends on both $f$ and $g$. The above estimate is a kind of absolute error analysis and is only in asymptotic sense. Typically, the constants $K$ and $c$ are quite complicated to estimate in practice.

Due to the computational cost of (1.2), people tend to choose small $d$ in practical applications. This is because (1.2) is very expensive to solve for big $d$ (the size of the resulting SDP grows exponentially in $d$ ). So, it is interesting to know how well (1.2) approximates (1.1) for a fixed relaxation order $d$. Suppose $\operatorname{deg}(f) \leqslant 2 d$ and $\operatorname{deg}(g) \leqslant 2 d$. For convenience, just denote by $f_{\text {sos }}$ the optimal value of (1.2) for a given $f$. We have seen that $f_{\text {sos }} \leqslant f_{\text {min }}$ but do not know how far away $f_{\text {sos }}$ is from $f_{\min }$. Denote by $f_{\max }$ the maximum of $f(x)$ on $S$, which always exists when $S$ is compact. For fixed $g$, a constant $Q=Q(g)$ is called an approximation bound of (1.2) if for every $f$ with $\operatorname{deg}(f) \leqslant 2 d$, it holds that

$$
f_{\max }-f_{\text {sos }} \leqslant Q \cdot\left(f_{\max }-f_{\min }\right) .
$$

Does the above $Q$ exist? What conditions make $Q$ exist? How big is $Q$ ? These questions are the main topics of this paper.

Contributions First, we analyze the approximation bound of Lasserre's relaxation (1.2) when $S$ is compact. Let $f_{\min }$ (resp., $f_{\max }$ ) be the minimum (resp., maximum) value of $f$ on $S$. Under a suitable condition on $g_{1}, \cdots, g_{m}$, we show that there exists a constant $Q=Q\left(g_{1}, \cdots, g_{m}\right)$ such that for every $f$ with $\operatorname{deg}(f) \leqslant 2 d$, it holds that

$$
1 \leqslant\left(f_{\max }-f_{\text {sos }}\right) /\left(f_{\max }-f_{\min }\right) \leqslant Q .
$$

The constant $Q$ only depends on $g_{1}, \cdots, g_{m}, n, d$ but not on $f$. This will be presented in Sect. 3.

Second, we give explicit estimates for $Q$ in (1.4) for special cases of $S$ : when $S$ is a unit ball, $Q=\mathcal{O}\left(n^{d}\right)$; when $S$ is a hypercube $[-1,1]^{n}, Q=\mathcal{O}\left(n^{2 d}\right)$; when $S$ is the boolean set $\{ \pm 1\}^{n}$ or $\{0,1\}^{n}, Q=\mathcal{O}\left(n^{d}\right)$; when $S$ is a multiunit ball, $Q=\mathcal{O}\left(n^{d}\right)$. (Here, we assume that canonical defining polynomials $g_{i}$ for $S$ are used.) This will be shown in Sect. 4.

The proofs of these approximation bounds are based on estimating norms of polynomials and using semidefinite programming properties. So, we will first introduce some basics about semidefinite programming, sum of squares, norms of polynomials, and their relations. This will be presented in Sect. 2 .

Here we make some remarks on the difference between estimates (1.3) and (1.4). The estimates for $Q$ given in this paper depend on $g$. As $d \rightarrow \infty$, the obtained bound $Q$ typically goes to infinity, which does not imply the convergence of (1.2). This is because the approximation bound and convergence are different aspects of Lasserre's relaxation. The bound $Q$ estimates $f_{\text {sos }}$ in the worst case that (1.2) would behave when the relaxation order $d$ is fixed. Estimate (1.4) holds for arbitrary polynomial $f$ 
of degree $2 d$ and can be thought of as a relative error analysis. The smaller $Q$ is, the tighter (1.2) approximates (1.1); the bigger $Q$ is, the looser (1.2) approximates (1.1). This is the reason why we call $Q$ an approximation bound. On the other hand, the convergence of (1.2) is the different issue of whether $f_{\text {sos, } d}$ approaches $f_{\min }$ as $d \rightarrow \infty$ for a fixed polynomial $f$. Estimate (1.3) holds for a fixed $f$ and can be thought of as an absolute error analysis. In convergence analysis, the polynomial $f$ in (1.1) is fixed, but the relaxation order $d$ goes to infinity. This issue is important when we want to minimize a fixed $f$ over $S$ as accurate as possible. In summary, the convergence concerns the behavior of a sequence of Lasserre's relaxations $(d=1,2, \cdots)$ for solving a fixed single polynomial optimization (1.1), while the approximation bound $Q$ concerns the behavior of a single Lasserre's relaxation (the order $d$ is fixed) for solving a class of polynomial optimization(1.1) ( $f$ is arbitrary with degree $2 d)$. So, $(1.3)$ and (1.4) address different aspects of Lasserre's relaxation.

Notation The symbol $\mathbb{N}$ (resp., $\mathbb{R}$ ) denotes the set of nonnegative integers (resp., real numbers). For any $t \in \mathbb{R},\lceil t\rceil$ (resp., $\lfloor t\rfloor$ ) denotes the smallest integer not smaller (resp., the largest integer not bigger) than $t$. For $0<k \in \mathbb{N},[k]=\{1, \cdots, k\}$. For $x \in \mathbb{R}^{n}, x_{i}$ denotes the $i$ th component of $x$, that is, $x=\left(x_{1}, \cdots, x_{n}\right)$. The symbol $\mathbb{S}^{n-1}$ denotes the unit sphere $\left\{x \in \mathbb{R}^{n}: x_{1}^{2}+\cdots+x_{n}^{2}=1\right\}$. For $\alpha \in \mathbb{N}^{n}$, denote $|\alpha|=$ $\alpha_{1}+\cdots+\alpha_{n}$ and $\operatorname{supp}(\alpha)=\left\{1 \leqslant i \leqslant n: \alpha_{i} \neq 0\right\}$. The symbol $\mathbb{N}_{k}^{n}$ denotes the multiindex set $\left\{\alpha \in \mathbb{N}^{n}:|\alpha| \leqslant k\right\}$. For $x \in \mathbb{R}^{n}$ and $\alpha \in \mathbb{N}^{n}, x^{\alpha}$ denotes $x_{1}^{\alpha_{1}} \cdots x_{n}^{\alpha_{n}}$. The symbol $[x]_{d}$ denotes the vector of monomials

$$
[x]_{d}^{T}=\left[\begin{array}{lllllllllllll}
1 & x_{1} & \cdots & x_{n} & x_{1}^{2} & x_{1} x_{2} & \cdots & \cdots & x_{1}^{d} & x_{1}^{d-1} x_{2} & \cdots & \cdots & x_{n}^{d}
\end{array}\right],
$$

and $\left[x^{d}\right]$ denotes the $d$ th homogeneous part of $[x]_{d}$, that is,

$$
\left[x^{d}\right]^{T}=\left[\begin{array}{lllll}
x_{1}^{d} & x_{1}^{d-1} x_{2} & \cdots & \cdots & x_{n}^{d}
\end{array}\right] .
$$

The symbol $\mathbb{R}[x]$ denotes the ring of real polynomials in $\left(x_{1}, \cdots, x_{n}\right) ; \mathbb{R}[x]_{k}$ denotes the subspace of polynomials of degrees at most $k$; $\operatorname{Sfr}[x]_{k}$ denotes the subspace of square-free polynomials of degrees at most $k$. For a polynomial $p, \operatorname{supp}(p)$ denotes the support of $p$, i.e., the set of $\alpha \in \mathbb{N}^{n}$ such that $x^{\alpha}$ appears in $p$. For a finite set $S,|S|$ denotes its cardinality; for a general set $S, \operatorname{int}(S)$ denotes its interior. For a matrix $A, A^{T}$ denotes its transpose. The symbol $I_{N}$ denotes the $N$-by- $N$ identity matrix. For a symmetric matrix $X, \lambda_{\max }(X)$ and $\lambda_{\min }(X)$ denote the maximum and minimum eigenvalues of $X$, respectively, and $X \succeq 0$ (resp., $X \succ 0$ ) means $\lambda_{\min }(X) \geqslant$ 0 (resp. $\lambda_{\min }(X)>0$ ). For $u \in \mathbb{R}^{N},\|u\|_{2}=\sqrt{u^{T} u}$ denotes the standard Euclidean norm. For any matrix $A$, recall the definition of $\|A\|_{2}$ being the standard operator 2-norm of $A$ and $\|A\|_{F}$ being the Frobenius norm of $A$, i.e., $\|A\|_{F}=\sqrt{\operatorname{Trace}\left(A^{T} A\right)}$. Note that $\|A\|_{2}$ and $\|A\|_{2} \leqslant\|A\|_{F}$. In matrix spaces, the bullet $\bullet$ denotes the standard Frobenius inner product, i.e., $A \bullet B=\operatorname{Trace}\left(A^{T} B\right)$.

\section{Sum of Squares and Norms of Polynomials}

This section presents some basics in sum of squares, semidefinite programming, norms of polynomials, and their relations. 


\subsection{Sum of Squares and Semidefinite Programming}

For a polynomial $f$ of degree $2 d$, there exists a symmetric matrix $F$ such that

$$
f(x)=[x]_{d}^{T} F[x]_{d} .
$$

The length of the monomial vector $[x]_{d}$ is $\left(\begin{array}{c}n+d \\ d\end{array}\right)$, and the dimension of $F$ is $\left(\begin{array}{c}n+d \\ d\end{array}\right) \times$ $\left(\begin{array}{c}n+d \\ d\end{array}\right)$. The matrix $F$ is called a Gram matrix of $f$ and is not unique if $d>1$. For convenience, we index the columns and rows of $F$ by monomials of degrees $\leqslant d$ or, equivalently, by vectors in $\mathbb{N}_{d}^{n}$.

A polynomial $f$ is said to be a sum of squares (SOS) if there exist polynomials $f_{1}, \cdots, f_{k} \in \mathbb{R}[x]$ such that $f=f_{1}^{2}+\cdots+f_{k}^{2}$. As shown in $[7,8], f$ is SOS if and only if it has a Gram matrix $F$ which is positive semidefinite, that is,

$$
f \text { is SOS } \Longleftrightarrow f(x)=[x]_{d}^{T} F[x]_{d} \text { for some matrix } F \succeq 0 .
$$

Define constant symmetric matrices $A_{\alpha}$ satisfying

$$
[x]_{d}[x]_{d}^{T}=\sum_{\alpha \in \mathbb{N}_{2 d}^{n}} A_{\alpha} x^{\alpha} .
$$

If $f(x)=\sum_{\alpha \in \mathbb{N}_{2 d}^{n}} f_{\alpha} x^{\alpha}$, then $f$ is SOS if and only if

$$
A_{\alpha} \bullet X=f_{\alpha} \quad \forall \alpha \in \mathbb{N}_{2 d}^{n} \text {, for some matrix } X \succeq 0 \text {. }
$$

So, checking whether $f$ is SOS can be done by solving a semidefinite program.

The standard form of a semidefinite program is

$$
\begin{cases}\min & C \bullet X \\ \text { s.t. } & A_{i} \bullet X=b_{i}, i=1, \cdots, m, \\ & X \succeq 0 .\end{cases}
$$

Here $C$ and $A_{1}, \cdots, A_{m}$ are constant symmetric matrices. Lasserre [2] showed that the SOS program (1.2) is equivalent to an SDP problem like (2.2). So (1.2) can be solved efficiently. SDP is a very nice convex optimization and has many attractive properties. There is a large amount of work on the theory, algorithms, and applications of semidefinite programming. We refer to [11].

\subsection{Norms of Polynomials}

For a polynomial $f(x)=\sum_{\alpha \in \mathbb{N}_{2 d}^{n}} f_{\alpha} x^{\alpha}$ of degree $2 d$, define its 2-norm and $G$-norm as

$$
\|f\|_{2}=\left(\sum_{\alpha \in \mathbb{N}_{2 d}^{n}} f_{\alpha}^{2}\right)^{1 / 2}, \quad\|f\|_{G}=\left(\sum_{\alpha \in \mathbb{N}_{2 d}^{n}} \mathfrak{p}(\alpha)^{-1} f_{\alpha}^{2}\right)^{1 / 2} .
$$


Here $\mathfrak{p}(\alpha)$ denotes the partition number of $\alpha$, that is,

$$
\mathfrak{p}(\alpha)=\left|\left\{(\beta, v) \in \mathbb{N}_{d}^{n} \times \mathbb{N}_{d}^{n}: \beta+v=\alpha\right\}\right| .
$$

Let $J$ be the matrix of all ones. Then

$$
\mathfrak{p}(\alpha)=A_{\alpha} \bullet J \leqslant\left(\begin{array}{c}
|\operatorname{supp}(\alpha)|+d \\
d
\end{array}\right) \leqslant\left(\begin{array}{c}
3 d \\
d
\end{array}\right) .
$$

Clearly, the norms $\|\cdot\|_{2}$ and $\|\cdot\|_{G}$ are equivalent and satisfy the relation

$$
\left(\begin{array}{c}
3 d \\
d
\end{array}\right)^{-1 / 2}\|f\|_{2} \leqslant\|f\|_{G} \leqslant\|f\|_{2} .
$$

In view of (2.3), for convenience, we denote the coefficient vectors

$$
f=\left(f_{\alpha}: \alpha \in \mathbb{N}_{2 d}^{n}\right), \quad f_{G}=\left(\mathfrak{p}(\alpha)^{-1 / 2} f_{\alpha}: \alpha \in \mathbb{N}_{2 d}^{n}\right),
$$

and denote by $[x]_{G, 2 d}$ the column vector of scaled monomials

$$
[x]_{G, 2 d}=\left(\mathfrak{p}(\alpha)^{1 / 2} x^{\alpha}: \alpha \in \mathbb{N}_{2 d}^{n}\right) .
$$

The entries of $f, f_{G}$, and $[x]_{G, 2 d}$ are in graded alphabetical ordering by their indices. Thus, $f(x)=f^{T}[x]_{2 d}=f_{G}^{T}[x]_{G, 2 d}$ and $\|f\|_{G}=\left\|f_{G}\right\|_{2}$. The $G$-norm $\|f\|_{G}$ is closely related to Gram matrices of $f$.

Lemma 2.1 If $f \in \mathbb{R}[x]_{2 d}$, there exists a symmetric matrix $W$ such that

$$
f(x)=[x]_{d}^{T} W[x]_{d}, \quad\|W\|_{F}=\|f\|_{G} .
$$

Proof For any matrix $W$ satisfying $f(x)=[x]_{d}^{T} W[x]_{d}$, it must hold that

$$
f_{\alpha}=\sum_{(\beta, v) \in \mathbb{N}_{d}^{n} \times \mathbb{N}_{d}^{n}: \beta+\nu=\alpha} W_{\beta, v} \quad \forall \alpha \in \mathbb{N}_{2 d}^{n} .
$$

Choose a particular $W$ satisfying the above as follows:

$$
W_{\beta, v}=\mathfrak{p}(\alpha)^{-1} f_{\alpha} \quad \forall(\beta, v) \in \mathbb{N}_{d}^{n} \times \mathbb{N}_{d}^{n}: \beta+v=\alpha .
$$

Then $W$ is symmetric and satisfies

$$
\|W\|_{F}^{2}=\sum_{\alpha \in \mathbb{N}_{2 d}^{n}} \sum_{\substack{(\beta, \nu) \in \mathbb{N}_{d}^{n} \times \mathbb{N}_{d}^{n} \\ \beta+\nu=\alpha}}\left(\mathfrak{p}(\alpha)^{-1} f_{\alpha}\right)^{2}=\sum_{\alpha \in \mathbb{N}_{2 d}^{n}}\left(\mathfrak{p}(\alpha)^{-1} f_{\alpha}\right)^{2} \mathfrak{p}(\alpha)=\|f\|_{G}^{2}
$$

This completes the proof.

Another useful norm of polynomials is the $L^{2}$-norm. Assume that $S$ is compact. Define

$$
\|f\|_{L^{2}(S)}:=\left(\int_{S} f(x)^{2} d \mu(x)\right)^{1 / 2}
$$


Here $\mu$ is the uniform probability measure on $S$. The $\|f\|_{L^{2}(S)}$ defined in (2.8) is a norm in $\mathbb{R}[x]$ when $S$ has nonempty interior. This is because if $\|f\|_{L^{2}(S)}=0$, then $f(x)$ vanishes in an open set, and it must be identically zero.

When $n \geqslant 2 d$, we can also define the following notion of marginal $L^{2}$-norm. Given a subset $\Delta \subset\{1, \cdots, n\}$ with $|\Delta|=2 d, x_{\Delta}$ denotes the subvector

$$
x_{\Delta}=\left(x_{i_{1}}, \cdots, x_{i_{2 d}}\right) \text { if } \Delta=\left\{i_{1}, \cdots, i_{2 d}\right\} .
$$

The restriction $f_{\Delta}\left(x_{\Delta}\right)$ of $f(x)$ to $x_{\Delta}$ is defined as

$$
f_{\Delta}\left(x_{\Delta}\right)=f(\tilde{x}), \quad \text { where } \tilde{x}_{i}= \begin{cases}x_{i} & \text { if } i \in \Delta, \\ 0 & \text { otherwise }\end{cases}
$$

So $f_{\Delta}\left(x_{\Delta}\right)$ is a polynomial in $x_{\Delta}$. Denote the set

$$
\Omega_{2 d}=\{\Delta \subset[n]:|\Delta|=2 d\} .
$$

Clearly, the cardinality $\left|\Omega_{2 d}\right|=\left(\begin{array}{c}n \\ 2 d\end{array}\right)$. We also denote by $g_{i, \Delta}\left(x_{\Delta}\right)$ the restriction of $g_{i, \Delta}(x)$ to $x_{\Delta}$. Define $S_{\Delta}$ similarly as

$$
S_{\Delta}=\left\{x_{\Delta}: g_{1, \Delta}\left(x_{\Delta}\right) \geqslant 0, \cdots, g_{m, \Delta}\left(x_{\Delta}\right) \geqslant 0\right\} .
$$

Observe that

$$
S_{\Delta}=\left\{x_{\Delta}:\left(0, \cdots, 0, x_{i_{1}}, 0, \cdots, 0, x_{i_{2 d}}, 0, \ldots\right) \in S\right\} \quad \text { if } \Delta=\left\{i_{1}, \cdots, i_{2 d}\right\} .
$$

If $0 \in \operatorname{int}(S)$, then every $S_{\Delta}$ has a nonempty interior, and $0 \in \operatorname{int}\left(S_{\Delta}\right)$. If $\operatorname{int}\left(S_{\Delta}\right) \neq \emptyset$, the $L^{2}\left(S_{\Delta}\right)$-norm of $f_{\Delta}\left(x_{\Delta}\right)$ can be similarly defined as

$$
\left\|f_{\Delta}\right\|_{L^{2}\left(S_{\Delta}\right)}=\left(\int_{S_{\Delta}} f_{\Delta}\left(x_{\Delta}\right)^{2} d \mu_{\Delta}\left(x_{\Delta}\right)\right)^{1 / 2},
$$

where $\mu_{\Delta}(\cdot)$ is the uniform probability measure on $S_{\Delta}$. When every $\operatorname{int}\left(S_{\Delta}\right) \neq \emptyset$, the marginal $L^{2}(S)$-norm of $f$ is defined as

$$
\|f\|_{L^{2}(S), m g}:=\left(\sum_{\Delta \in \Omega_{2 d}}\left\|f_{\Delta}\right\|_{L^{2}\left(S_{\Delta}\right)}^{2}\right)^{1 / 2} .
$$

Define two matrices

$$
\begin{aligned}
\boldsymbol{\Theta}_{\Delta, 2 d}(S) & =\int_{S_{\Delta}}\left[x_{\Delta}\right]_{G, 2 d}\left[x_{\Delta}\right]_{G, 2 d}^{T} d \mu_{\Delta}\left(x_{\Delta}\right), \\
\boldsymbol{\Theta}_{2 d}(S) & =\int_{S}[x]_{G, 2 d}[x]_{G, 2 d}^{T} d \mu(x),
\end{aligned}
$$

and two constants associated to $S$

$$
\kappa_{2 d}(S)=\min _{p \in \mathbb{R}[x]_{2 d}}\left\{\|p\|_{L^{2}(S)}:\|p\|_{G}=1\right\},
$$




$$
\eta_{2 d}(S)=\sqrt{\min _{\Delta \in \Omega_{2 d}} \lambda_{\min }\left(\boldsymbol{\Theta}_{\Delta, 2 d}(S)\right)}
$$

If we write $p(x)=p_{G}^{T}[x]_{G, 2 d}$, then

$$
\|p\|_{L^{2}(S)}^{2}=p_{G}^{T} \boldsymbol{\Theta}_{2 d}(S) p_{G}
$$

So, $\kappa_{2 d}(S)=\sqrt{\lambda_{\min }\left(\boldsymbol{\Theta}_{2 d}(S)\right)}$. These constants depend only on $S$, and there are explicit formulae for them when $S$ is special, like a ball or hypercube.

Lemma 2.2 If $\operatorname{int}\left(S_{\Delta}\right) \neq \emptyset$ for every $\Delta \in \Omega_{2 d}$, then $\eta_{2 d}(S)>0$. In particular, if $0 \in \operatorname{int}(S)$, then $\eta_{2 d}(S)>0$ and $\kappa_{2 d}(S)>0$.

Proof It suffices to prove that every $\boldsymbol{\Theta}_{\Delta, 2 d}(S)$ is positive definite. Otherwise, suppose that there exists $u \neq 0$ such that $u^{T} \boldsymbol{\Theta}_{\Delta, 2 d}(S) u=0$ for some $\Delta \in \Omega_{2 d}$. Then

$$
u^{T} \boldsymbol{\Theta}_{\Delta, 2 d}(S) u=\int_{S_{\Delta}}\left(u^{T}\left[x_{\Delta}\right]_{G, 2 d}\right)^{2} d \mu_{\Delta}\left(x_{\Delta}\right)=0
$$

implies $u^{T}\left[x_{\Delta}\right]_{G, 2 d}=0$ for every $x \in S_{\Delta}$. Since $\operatorname{int}\left(S_{\Delta}\right) \neq \emptyset, u^{T}\left[x_{\Delta}\right]_{G, 2 d}$ must be identically zero, and $u=0$, which is a contradiction.

If $0 \in \operatorname{int}(S)$, then $0 \in \operatorname{int}\left(S_{\Delta}\right)$ for every $\Delta$. Thus, $\eta_{2 d}(S)>0$ follows from the above. The proof of $\kappa_{2 d}(S)>0$ is also the same.

The norms $\|\cdot\|_{L^{2}(S), m g}$ and $\|\cdot\|_{G}$ are related by the following lemma.

Lemma 2.3 Suppose $n \geqslant 2 d$. If $f \in \mathbb{R}[x]_{2 d}$, then

$$
\|f\|_{L^{2}(S), m g} \geqslant \eta_{2 d}(S)\|f\|_{G} .
$$

Proof By the definitions of $L^{2}\left(S_{\Delta}\right)$-norm and $\eta_{2 d}(S)$, we know that

$$
\left\|f_{\Delta}\right\|_{L^{2}\left(S_{\Delta}\right)}^{2}=f_{\Delta, G}^{T} \boldsymbol{\Theta}_{\Delta, 2 d}(S) f_{\Delta, G} \geqslant \eta_{2 d}(S)^{2}\left\|f_{\Delta}\right\|_{G}^{2} .
$$

Here, $f_{\Delta, G}$ denotes the vector of scaled coefficients of $f_{\Delta}$ (see (2.6)). So

$$
\|f\|_{L^{2}(S), m g}^{2}=\sum_{\Delta \in \Omega_{2 d}}\left\|f_{\Delta}\right\|_{L^{2}\left(S_{\Delta}\right)}^{2} \geqslant \eta_{2 d}(S)^{2} \sum_{\Delta \in \Omega_{2 d}}\left\|f_{\Delta}\right\|_{G}^{2} \geqslant \eta_{2 d}(S)^{2}\|f\|_{G}^{2} .
$$

Thus, the lemma follows.

\section{Some General Bounds for Lasserre's Relaxation}

This section estimates the approximation bound of Lasserre's relaxation (1.2). As mentioned in Introduction, to make Lasserre's relaxations converge, one needs to assume the archimedean condition (AC). However, even if AC holds, it is still possible 
that (1.2) is infeasible (i.e., $f_{\text {sos }}=-\infty$ ) for a given $d$, though an arbitrarily good lower bound could be obtained if we increase the relaxation order. To guarantee the feasibility of (1.2), we need the following assumption.

Assumption 3.1 There exist a symmetric positive definite matrix $E$ and SOS polynomials $\sigma_{1}, \cdots, \sigma_{m}$ such that $\operatorname{deg}\left(\sigma_{i} g_{i}\right) \leqslant 2 d$ for every $i$ and

$$
\sigma_{1} g_{1}+\cdots+\sigma_{m} g_{m}=1-[x]_{d}^{T} E[x]_{d} .
$$

Note that Assumption 3.1 is equivalent to that the constant polynomial 1 lies in the interior of the truncated quadratic module defined as

$$
M_{2 d}\left(g_{1}, \cdots, g_{m}\right)=\left\{\sigma_{0}+\sum_{j=1}^{m} \sigma_{j} g_{j}: \text { each } g_{j} \text { is SOS, and } \operatorname{deg}\left(\sigma_{j} g_{j}\right) \leqslant 2 d\right\} \text {. }
$$

Assumption 3.1 is sufficient and necessary for (1.2) to have a finite optimal value for every $f \in \mathbb{R}[x]_{2 d}$.

Proposition 3.2 Suppose $S$ is nonempty. Then the following statements are equivalent:

(i) Assumption 3.1 holds;

(ii) The relaxation (1.2) is feasible for every $f \in \mathbb{R}[x]_{2 d}$;

(iii) The relaxation (1.2) is feasible for $f=-[x]_{d}^{T}[x]_{d}$.

Proof We first prove (i) $\Rightarrow$ (ii). Every $f \in \mathbb{R}[x]_{2 d}$ can be written as $f(x)=$ $[x]_{d}^{T} F[x]_{d}$ for some symmetric matrix $F$. By Assumption 3.1, the matrix $E$ is positive definite, so we can choose $\lambda>0$ big enough such that the polynomial

$$
\sigma_{0}(x):=f(x)+\lambda[x]_{d}^{T} E[x]_{d}=[x]_{d}^{T}(F+\lambda E)[x]_{d}
$$

is SOS. Then choose $\gamma=-\lambda$, and we get the identity

$$
f-\gamma=\sigma_{0}+\lambda \sigma_{1} g_{1}+\cdots+\lambda \sigma_{m} g_{m}
$$

Therefore, (1.2) is feasible, and its optimal value $f_{\text {sos }} \geqslant \gamma$.

The direction (ii) $\Rightarrow$ (iii) is obvious. Now we prove (iii) $\Rightarrow$ (i). Let $f(x)=$ $-[x]_{d}^{T}[x]_{d}$. Since (1.2) is feasible for this $f$, there exist $\hat{\gamma}$ and SOS polynomials $\hat{\sigma}_{0}, \hat{\sigma}_{1}, \cdots, \hat{\sigma}_{m}$ such that $\operatorname{deg}\left(\hat{\sigma}_{i} g_{i}\right) \leqslant 2 d$ for every $i$ and

$$
-[x]_{d}^{T}[x]_{d}-\hat{\gamma}=\hat{\sigma}_{0}+\hat{\sigma}_{1} g_{1}+\cdots+\hat{\sigma}_{m} g_{m} .
$$

Evaluating at $u \in S$, we obtain that $-\hat{\gamma} \geqslant[u]_{d}^{T}[u]_{d} \geqslant 1$ and

$$
\frac{1}{-\hat{\gamma}}\left(\hat{\sigma}_{1} g_{1}+\cdots+\hat{\sigma}_{m} g_{m}\right)=1-\frac{1}{-\hat{\gamma}}\left([x]_{d}^{T}[x]_{d}+\hat{\sigma}_{0}\right) .
$$

Hence, Assumption 3.1 holds. 
In Assumption 3.1, the choice of SOS polynomials $\sigma_{1}, \cdots, \sigma_{m}$ and positive definite matrix $E$ might not be unique. In our bound analysis, the bigger $\lambda_{\min }(E)$ is, the better the obtained bound is. So we want $\lambda_{\min }(E)$ to be as large as possible. Interestingly, the best one could be found by solving the following SOS program:

$$
\begin{cases}\max _{\sigma_{1}, \cdots, \sigma_{m}, E} & \lambda_{\min }(E) \\ \text { s.t. } & \sigma_{1} g_{1}+\cdots+\sigma_{m} g_{m}=1-[x]_{d}^{T} E[x]_{d}, \\ & \sigma_{1}, \cdots, \sigma_{m} \operatorname{are~SOS,} \\ & \operatorname{deg}\left(\sigma_{1} g_{1}\right), \cdots, \operatorname{deg}\left(\sigma_{m} g_{m}\right) \leqslant 2 d .\end{cases}
$$

Assume that $\left(\sigma_{1}^{*}, \cdots, \sigma_{m}^{*}, E^{*}\right)$ is optimal for (3.1). Assumption 3.1 holds if and only if $\lambda_{\min }\left(E^{*}\right)>0$, so it is checkable by solving the SOS program (3.1).

Let $d=\max \{\lceil\operatorname{deg}(f) / 2\rceil,\lceil\operatorname{deg}(g) / 2\rceil\}$, and $\mathcal{F}$ be a subspace of $\mathbb{R}[x]_{2 d}$ containing $f$. Define a constant associated with $\mathcal{F}$ and $S$ as

$$
\chi(\mathcal{F}, S):=\max _{p \in \mathcal{F}}\left\{\|p\|_{G}:|p(x)| \leqslant 1 \forall x \in S\right\} .
$$

When $\operatorname{int}(S) \neq \emptyset, \chi(\mathcal{F}, S)<\infty$ because $\kappa_{2 d}(S)>0($ cf. Lemma 2.2) and

$$
\|p\|_{L^{2}(S)} \geqslant \kappa_{2 d}(S) \cdot\left\|p_{G}\right\|_{2} .
$$

When $\operatorname{int}(S)=\emptyset, \chi(\mathcal{F}, S)$ might be infinite for some $\mathcal{F}$. For instance, if $S$ is the unit sphere $\mathbb{S}^{n-1}$, then $\chi\left(\mathbb{R}[x]_{2 d}, \mathbb{S}^{n-1}\right)=\infty$ because for $p_{k}=k\left(1-\|x\|_{2}^{2}\right)$, it holds that

$$
\left\|p_{k}\right\|_{G} \rightarrow \infty \text { as } k \rightarrow \infty \quad \text { while }\left|p_{k}(x)\right| \leqslant 1 \quad \forall x \in \mathbb{S}^{n-1}
$$

When $S$ has an empty interior, in order to ensure $\chi(\mathcal{F}, S)<\infty$, it suffices to choose $\mathcal{F}$ lying in the orthogonal complement of the subspace space

$$
\mathcal{V}(S)=\left\{p \in \mathbb{R}[x]_{2 d}: p(x)=0 \forall x \in S\right\} .
$$

Obviously, if $\operatorname{int}(S) \neq \emptyset, \mathcal{V}(S)=\{0\}$ is a singleton.

Theorem 3.3 Suppose that $\mathcal{F}$ is a subspace of $\mathbb{R}[x]_{2 d}$ containing $1, \chi(\mathcal{F}, S)<\infty$, and the tuple $\left(\sigma_{1}, \cdots, \sigma_{m}, E\right)$ satisfies Assumption 3.1. Let $f \in \mathcal{F}$, and $f_{\min }$ (resp. $f_{\max }$ ) be its minimum (resp. maximum) on $S$. If $f_{\mathrm{sos}}$ is the optimal value of (1.2), then

$$
1 \leqslant \frac{f_{\max }-f_{\text {sos }}}{f_{\max }-f_{\min }} \leqslant \frac{\chi(\mathcal{F}, S)}{\lambda_{\min }(E)} .
$$

The above bound is best if the optimal solution $\left(\sigma_{1}^{*}, \cdots, \sigma_{m}^{*}, E^{*}\right)$ of $(3.1)$ is used.

Proof Let $\operatorname{med}(f)=\frac{1}{2}\left(f_{\min }+f_{\max }\right) \in\left[f_{\min }, f_{\max }\right]$. First, we consider the case that $f_{\min }<\operatorname{med}(f)<f_{\max }$. Let

$$
\tilde{f}(x):=\frac{f(x)-\operatorname{med}(f)}{\operatorname{med}(f)-f_{\min }} \in \mathcal{F} .
$$


Then, $|\tilde{f}(x)| \leqslant 1$ for all $x \in S$, and $\|\tilde{f}\|_{G} \leqslant \chi(\mathcal{F}, S)$ by definition (3.2). Now set

$$
\theta^{*}:=\frac{\chi(\mathcal{F}, S)}{\lambda_{\min }(E)}>0, \quad \gamma^{*}:=\operatorname{med}(f)-\theta^{*}\left(\operatorname{med}(f)-f_{\min }\right) .
$$

Then, we have

$$
\left\|\frac{1}{\theta^{*}} \tilde{f}\right\|_{G} \leqslant \lambda_{\min }(E) .
$$

By Lemma 2.1, there exists a symmetric matrix $W$ satisfying

$$
\begin{gathered}
\frac{1}{\theta^{*}} \tilde{f}(x)=[x]_{d}^{T} W[x]_{d}, \quad\|W\|_{F} \leqslant \lambda_{\min }(E), \\
\frac{1}{\theta^{*}} \tilde{f}(x)+[x]_{d}^{T} E[x]_{d}=[x]_{d}^{T}(W+E)[x]_{d} .
\end{gathered}
$$

Since $\|W\|_{2} \leqslant\|W\|_{F} \leqslant \lambda_{\min }(E)$, we know that $W+E \succeq 0$. Hence, the polynomial

$$
\hat{\sigma}_{0}(x):=\left(\operatorname{med}(f)-f_{\min }\right) \theta^{*}\left(\frac{1}{\theta^{*}} \tilde{f}(x)+[x]_{d}^{T} E[x]_{d}\right)
$$

must be SOS. Let $\hat{\sigma}_{i}=\left(\operatorname{med}(f)-f_{\min }\right) \theta^{*} \sigma_{i}$ for every $i$, which are all SOS. Then we can verify that

$$
\begin{aligned}
\hat{\sigma}_{1} g_{1}+\cdots+\hat{\sigma}_{m} g_{m} & =\left(\operatorname{med}(f)-f_{\min }\right) \theta^{*}\left(1-[x]_{d}^{T} E[x]_{d}\right), \\
f-\gamma^{*} & =\hat{\sigma}_{0}+\hat{\sigma}_{1} g_{1}+\cdots+\hat{\sigma}_{m} g_{m} .
\end{aligned}
$$

So, $\left(\hat{\sigma}_{0}, \hat{\sigma}_{1}, \cdots, \hat{\sigma}_{m}, \gamma^{*}\right)$ is feasible for (1.2), and its optimal value $f_{\text {sos }} \geqslant \gamma^{*}$. By the choice of $\gamma^{*}$ in (3.3), it holds that

$$
\frac{\operatorname{med}(f)-f_{\text {sos }}}{\operatorname{med}(f)-f_{\min }} \leqslant \frac{\chi(\mathcal{F}, S)}{\lambda_{\min }(E)} .
$$

Since $\operatorname{med}(f) \in\left[f_{\min }, f_{\max }\right]$ and $f_{\text {sos }} \leqslant f_{\min }$, the theorem is true.

Second, we consider the case that $f_{\min }=f_{\max }$; then $f-f_{\min }$ is constantly zero on $S$. Since $\chi(\mathcal{F}, S)<\infty, f-f_{\min }$ must be the identically zero polynomial, i.e., $f$ is the constant $f_{\min }$. So, $f_{\mathrm{sos}}=f_{\min }$, and the theorem is clearly true.

To get a concrete bound by applying Theorem 3.3, we need to estimate $\chi(\mathcal{F}, S)$ and $\lambda_{\min }(E)$.

Theorem 3.4 Suppose that $0 \in \operatorname{int}(S)$ and $\left(\sigma_{1}, \cdots, \sigma_{m}, E\right)$ satisfies Assumption 3.1. Let $f \in \mathbb{R}[x]_{2 d}, f_{\min }$ (resp. $f_{\max }$ ) be its minimum (resp. maximum) on $S$, and $f_{\mathrm{sos}}$ be the optimal value of Lasserre's relaxation (1.2).

(i) It holds that

$$
1 \leqslant \frac{f_{\max }-f_{\text {sos }}}{f_{\max }-f_{\min }} \leqslant \frac{1}{\kappa_{2 d}(S) \lambda_{\min }(E)} .
$$


(ii) If $n \geqslant 2 d$, then

$$
1 \leqslant \frac{f_{\max }-f_{\text {sos }}}{f_{\max }-f_{\min }} \leqslant \frac{1}{\eta_{2 d}(S) \lambda_{\min }(E)} \sqrt{\left(\begin{array}{c}
n \\
2 d
\end{array}\right)} .
$$

The above bounds are best if the optimal solution $\left(\sigma_{1}^{*}, \cdots, \sigma_{m}^{*}, E^{*}\right)$ of (3.1) is used.

Proof (i) Since $\operatorname{int}(S) \neq \emptyset$, Lemma 2.2 implies $\kappa_{2 d}(S)>0$. Let $\mathcal{F}=\mathbb{R}[x]_{2 d}$. If $p \in \mathcal{F}$ satisfies $|p(x)| \leqslant 1$ for all $x \in S$, then $\|p\|_{L^{2}(S)} \leqslant 1$, and (2.14) implies $1 \geqslant \kappa_{2 d}(S)\|p\|_{G}$ and $\chi(\mathcal{F}, S) \leqslant \frac{1}{\kappa_{2 d}(S)}$. Then, Theorem 3.3 implies the result.

(ii) Since $0 \in \operatorname{int}(S)$, every $S_{\Delta}$ has a nonempty interior, and Lemma 2.2 implies $\eta_{2 d}(S)>0$. If $p \in \mathbb{R}[x]_{2 d}$ and $|p(x)| \leqslant 1$ for all $x \in S$, the restriction $p_{\Delta}\left(x_{\Delta}\right)$ of $p(x)$ must also satisfy $\left|p_{\Delta}\left(x_{\Delta}\right)\right| \leqslant 1$ for every $x_{\Delta} \in S_{\Delta}$. Thus, we have

$$
\|p\|_{L^{2}(S), m g}^{2}=\sum_{\Delta \in \Omega_{2 d}} \int_{S_{\Delta}} p_{\Delta}\left(x_{\Delta}\right)^{2} d \mu_{\Delta}\left(x_{\Delta}\right) \leqslant \sum_{\Delta \in \Omega_{2 d}} 1=\left(\begin{array}{c}
n \\
2 d
\end{array}\right) .
$$

Therefore, Lemma 2.3 and the above imply

$$
\|p\|_{G} \leqslant \frac{1}{\eta_{2 d}(S)} \sqrt{\left(\begin{array}{c}
n \\
2 d
\end{array}\right)} \quad \text { and } \quad \chi\left(\mathbb{R}[x]_{2 d}, S\right) \leqslant \frac{1}{\eta_{2 d}(S)} \sqrt{\left(\begin{array}{c}
n \\
2 d
\end{array}\right)} .
$$

The result is then implied by Theorem 3.3.

Generally, it is hard to tell which one of $\frac{1}{\kappa_{2 d}(S)}$ and $\frac{1}{\eta_{2 d}(S)} \sqrt{\left(\begin{array}{c}n \\ 2 d\end{array}\right)}$ is superior in Theorem 3.4, depending on $S$. However, typically $\eta_{2 d}(S)$ is relatively easier to estimate than $\kappa_{2 d}(S)$ does. For instance, when $S$ is a unit ball or hypercube, the constant $\eta_{2 d}(S)$ is independent of $n$ and easy to estimate, while $\kappa_{2 d}(S)$ is quite difficult to estimate in terms of $n, d$. This will be shown in Sect. 4 .

Remark 3.5 The optimal value $\lambda_{\min }\left(E^{*}\right)$ of (3.1) is closely related to the "radius" of $S$. Let $R=\max _{x \in S}\|x\|_{2}$. Observe that

$$
\begin{gathered}
\|x\|_{2}^{2 k}=\left(x_{1}^{2}+\cdots+x_{n}^{2}\right)^{k}=\sum_{\alpha \in \mathbb{N}_{=k}^{n}} x^{2 \alpha} \frac{k !}{\alpha_{1} ! \cdots \alpha_{n} !} \leqslant k ! \sum_{\alpha \in \mathbb{N}_{=k}^{n}} x^{2 \alpha}=k !\left\|\left[x^{k}\right]\right\|_{2}^{2}, \\
\left\|[x]_{d}\right\|_{2}^{2}=1+\left\|\left[x^{1}\right]\right\|_{2}^{2}+\left\|\left[x^{2}\right]\right\|_{2}^{2}+\cdots+\left\|\left[x^{d}\right]\right\|_{2}^{2} \geqslant \sum_{k=0}^{d} \frac{\|x\|_{2}^{2 k}}{k !} .
\end{gathered}
$$

Since $1 \geqslant[x]_{d}^{T} E^{*}[x]_{d} \geqslant \lambda_{\min }\left(E^{*}\right)\left\|[x]_{d}\right\|_{2}^{2}$ for all $x \in S$, the above implies

$$
\frac{1}{\lambda_{\min }\left(E^{*}\right)} \geqslant \sum_{k=0}^{d} \frac{R^{2 k}}{k !}
$$


On the other hand, from

$$
\left\|\left[x^{k}\right]\right\|_{2}^{2}=\sum_{\alpha \in \mathbb{N}_{=k}^{n}} x^{2 \alpha} \leqslant \sum_{\alpha \in \mathbb{N}_{=k}^{n}} x^{2 \alpha} \frac{k !}{\alpha_{1} ! \cdots \alpha_{n} !}=\left(x_{1}^{2}+\cdots+x_{n}^{2}\right)^{k}=\|x\|_{2}^{2 k}
$$

we know that the polynomial $r(x):=1-\left(1+R^{2}+\cdots+R^{2 d}\right)^{-1}[x]_{d}^{T}[x]_{d}$ is nonnegative on $S$. If there exist SOS polynomials $s_{0}, s_{1}, \cdots, s_{m}$ such that every $\operatorname{deg}\left(s_{i} g_{i}\right) \leqslant$ $2 d$ and

$$
r=s_{0}+s_{1} g_{1}+\cdots+s_{m} g_{m},
$$

then we can find a positive definite matrix $\hat{E}$ satisfying

$$
[x]_{d}^{T} \hat{E}[x]_{d}=1-\left(s_{1} g_{1}+\cdots+s_{m} g_{m}\right)=s_{0}+\left(1+R^{2}+\cdots+R^{2 d}\right)^{-1}[x]_{d}^{T}[x]_{d} .
$$

Since $s_{0}$ is SOS, we know that

$$
\frac{1}{\lambda_{\min }\left(E^{*}\right)} \leqslant \frac{1}{\lambda_{\min }(\hat{E})} \leqslant \sum_{k=0}^{d} R^{2 k} \leqslant d ! \sum_{k=0}^{d} \frac{R^{2 k}}{k !} .
$$

So, if (3.5) holds, then

$$
\sum_{k=0}^{d} \frac{R^{2 k}}{k !} \leqslant \frac{1}{\lambda_{\min }\left(E^{*}\right)} \leqslant d ! \sum_{k=0}^{d} \frac{R^{2 k}}{k !}
$$

If (3.5) fails but $R$ is known in advance, we can add to (1.1) the redundant constraint $r(x) \geqslant 0$. Hence, $\frac{1}{\lambda_{\min }\left(E^{*}\right)}$ can be estimated by $\sum_{k=0}^{d} \frac{R^{2 k}}{k !}$, which is tight within a factor of the constant $d !$.

\section{Bounds for Some Special Optimization Problems}

Theorems 3.3 and 3.4 give some general bounds for Lasserre's relaxation (1.2) in terms of some constants related to the feasible set $S$. For special $S$ like a unit ball or hypercube, estimating $\eta_{2 d}(S)$ is typically easy, while estimating $\kappa_{2 d}(S)$ in terms of $n, d$ is typically quite difficult. So, generally we apply Theorem 3.4(ii) by estimating $\eta_{2 d}(S)$. In this section, we assume that $\operatorname{deg}(f)=2 d$ and $\operatorname{deg}\left(g_{i}\right)=2$.

\subsection{Optimizing Polynomials over a Unit Ball}

Consider the case that $S$ is the unit ball $\mathbf{B}:=\left\{x \in \mathbb{R}^{n}:\|x\|_{2} \leqslant 1\right\}$. Then $m=1$ and $g_{1}(x)=1-\|x\|_{2}^{2}$. To get a bound, we estimate $\eta_{2 d}(\mathbf{B})$ and $\lambda_{\min }\left(E^{*}\right)$ in (3.1).

By definition (2.15), we have $\eta_{2 d}(\mathbf{B})=\sqrt{\lambda_{\min }\left(\boldsymbol{\Theta}_{\Delta, 2 d}(\mathbf{B})\right)}$ for every $\Delta \in \Omega_{2 d}$ because of the symmetry of $\mathbf{B}$. Let $\Delta=\{1, \cdots, 2 d\}$; then 
Table 1 A list of $\eta_{2 d}(\mathbf{B})$ for $2 d=2,4,6,8$

\begin{tabular}{lllll}
\hline $2 d$ & 2 & 4 & 6 & 8 \\
\hline$\eta_{2 d}(\mathbf{B})$ & 0.19204 & 0.01670 & 0.00161 & 0.00004 \\
\hline
\end{tabular}

$$
\begin{aligned}
\boldsymbol{\Theta}_{\Delta, 2 d}(\mathbf{B}) & =\int_{\left\|x_{\Delta}\right\|_{2} \leqslant 1}\left[x_{\Delta}\right]_{G, 2 d}\left[x_{\Delta}\right]_{G, 2 d}^{T} d \mu_{\Delta}\left(x_{\Delta}\right) \\
& =\frac{1}{\operatorname{Vol}\left(\left\|x_{\Delta}\right\|_{2} \leqslant 1\right)} \int_{\left\|x_{\Delta}\right\|_{2} \leqslant 1}\left[x_{\Delta}\right]_{G, 2 d}\left[x_{\Delta}\right]_{G, 2 d}^{T} d x_{\Delta},
\end{aligned}
$$

where $d x_{\Delta}$ is the standard Lebesgue measure. Observe that

$$
\int_{\left\|x_{\Delta}\right\|_{2} \leqslant 1} x_{\Delta}^{\alpha} d x_{\Delta}=\operatorname{Area}\left(\mathbb{S}^{2 d-1}\right) \cdot \int_{\left\|x_{\Delta}\right\|_{2}=1} x_{\Delta}^{\alpha} d v_{\Delta}\left(x_{\Delta}\right) \cdot \int_{0}^{1} r^{|\alpha|+2 d-1} d r .
$$

In the above, $\operatorname{Area}\left(\mathbb{S}^{2 d-1}\right)$ is the area of the unit sphere $\mathbb{S}^{2 d-1}$, and $v_{\Delta}(\cdot)$ is the uniform probability measure on $\mathbb{S}^{2 d-1}$. Note the formulae

$$
\operatorname{Area}\left(\mathbb{S}^{2 d-1}\right)=\frac{2 \pi^{d}}{\Gamma(d)}, \quad \operatorname{Vol}\left(\left\|x_{\Delta}\right\|_{2} \leqslant 1\right)=\frac{\pi^{d}}{\Gamma(1+d)} .
$$

When $\alpha=2 \beta=2\left(\beta_{1}, \cdots, \beta_{2 d}\right)$ is an even vector, it holds that (cf. Lemma 8 of [1])

$$
\begin{aligned}
\int_{\left\|x_{\Delta}\right\|_{2}=1} x_{\Delta}^{\alpha} d v_{\Delta}\left(x_{\Delta}\right) & =\frac{\Gamma(d) \prod_{i=1}^{2 d} \Gamma\left(\beta_{i}+1 / 2\right)}{\pi^{d} \Gamma(|\beta|+d)}, \\
\int_{\left\|x_{\Delta}\right\|_{2} \leqslant 1} x_{\Delta}^{\alpha} d \mu_{\Delta}\left(x_{\Delta}\right) & =\frac{\Gamma(1+d) \prod_{i=1}^{2 d} \Gamma\left(\beta_{i}+1 / 2\right)}{\pi^{d}(|\beta|+d) \Gamma(|\beta|+d)} .
\end{aligned}
$$

Here, $\Gamma(\cdot)$ is the standard Gamma function. If at least one entry of $\alpha$ is odd, the integral $\int_{\left\|x_{\Delta}\right\|_{2}=1} x_{\Delta}^{\alpha} d v_{\Delta}\left(x_{\Delta}\right)=0$. A list of typical values of $\eta_{2 d}(\mathbf{B})$ is given in Table 1 .

Lemma 4.1 When $S$ is the unit ball $\mathbf{B}$, we have that $\eta_{2 d}(\mathbf{B})$ is independent of $n$, Assumption 3.1 holds, and the optimal $E^{*}$ of (3.1) satisfies $\lambda_{\min }\left(E^{*}\right) \geqslant \frac{1}{d+1}$.

Proof In the above, we have already seen that $\eta_{2 d}(\mathbf{B})$ is independent of $n$. Now we estimate $\lambda_{\min }\left(E^{*}\right)$ in (3.1). For any integer $k \geqslant 1$, it holds that

$$
\left(1+t+\cdots+t^{k-1}\right)(1-t)=1-t^{k} .
$$

Let $s_{d}(t):=\frac{1}{d+1} \sum_{k=1}^{d} \sum_{j=0}^{k-1} t^{j} ;$ then

$$
s_{d}(t)(1-t)=1-\frac{1}{d+1}\left(1+t+\cdots+t^{d}\right) .
$$

Plugging $t$ by $\|x\|_{2}^{2}$, we get

$$
s_{d}\left(\|x\|_{2}^{2}\right)\left(1-\|x\|_{2}^{2}\right)=1-\frac{1}{d+1}\left(1+\|x\|_{2}^{2}+\cdots+\|x\|_{2}^{2 d}\right) .
$$


Table 2 A list of $\eta_{2 d}(\mathcal{C})$ for $2 d=2,4,6,8$

\begin{tabular}{lllll}
\hline $2 d$ & 2 & 4 & 6 & 8 \\
\hline$\eta_{2 d}(\mathcal{C})$ & 0.2678 & 0.0671 & 0.0329 & 0.0096 \\
\hline
\end{tabular}

Since $s_{d}\left(\|x\|_{2}^{2}\right)$ is SOS and has degree $2 d-2$, there exists a symmetric $E$ satisfying

$$
\frac{1}{d+1}\left(1+\|x\|_{2}^{2}+\cdots+\|x\|_{2}^{2 d}\right)=[x]_{d}^{T} E[x]_{d}, \quad \lambda_{\min }(E) \geqslant \frac{1}{d+1} .
$$

So, Assumption 3.1 holds, and the optimal value of (3.1) is at least $\frac{1}{d+1}$.

Clearly, Theorem 3.4(ii) and Lemma 4.1 imply the following.

Corollary 4.2 Assume that $n \geqslant 2 d$. Let $f \in \mathbb{R}[x]_{2 d}$, and $f_{\min }$ (resp., $f_{\max }$ ) be its minimum (resp., maximum) on the unit ball B. If $f_{\text {sos }}$ is the optimal value of (1.2), then

$$
1 \leqslant \frac{f_{\max }-f_{\mathrm{sos}}}{f_{\max }-f_{\min }} \leqslant \frac{d+1}{\eta_{2 d}(\mathbf{B})} \sqrt{\left(\begin{array}{c}
n \\
2 d
\end{array}\right)} .
$$

So, $f_{\mathrm{sos}}$ is an $\mathcal{O}\left(n^{d}\right)$-approximation of $f_{\min }$ as $n \rightarrow \infty$.

\subsection{Optimizing Polynomials over a Hypercube}

Consider the case that $S$ is the hypercube $\mathcal{C}:=[-1,1]^{n}$. Then $m=n$ and

$$
g_{1}(x)=1-x_{1}^{2}, \cdots, g_{n}(x)=1-x_{n}^{2} .
$$

To get a bound by Theorem 3.4(ii), we need to estimate $\eta_{2 d}(\mathcal{C})$ and $\lambda_{\min }\left(E^{*}\right)$ in (3.1).

By definition (2.15), we know that $\eta_{2 d}(\mathcal{C})=\sqrt{\lambda_{\min }\left(\Theta_{\Delta, 2 d}(\mathcal{C})\right)}$ for every $\Delta \in \Omega_{2 d}$ since $\mathcal{C}$ is symmetric. Let $\Delta=\{1, \cdots, 2 d\}$, then

$$
\boldsymbol{\Theta}_{\Delta, 2 d}(\mathcal{C})=\int_{[-1,1]^{2 d}}\left[x_{\Delta}\right]_{G, 2 d}\left[x_{\Delta}\right]_{G, 2 d}^{T} d \mu_{\Delta}\left(x_{\Delta}\right) .
$$

If at least one $\alpha_{i}$ is odd, the integral $\int_{[-1,1]^{2 d}} x_{\Delta}^{\alpha} d \mu_{\Delta}\left(x_{\Delta}\right)$ vanishes. If $\alpha=$ $2\left(\beta_{1}, \cdots, \beta_{2 d}\right)$ is even, then

$$
\int_{[-1,1]^{2 d}} x_{\Delta}^{\alpha} d \mu_{\Delta}\left(x_{\Delta}\right)=\frac{1}{\left(1+2 \beta_{1}\right) \cdots\left(1+2 \beta_{2 d}\right)} .
$$

A list of typical values of $\eta_{2 d}(\mathcal{C})$ is in Table 2.

Lemma 4.3 When $S$ is the hypercube $\mathcal{C}=[-1,1]^{n}$, we have that $\eta_{2 d}(\mathcal{C})$ is independent of $n$, Assumption 3.1 holds, and the optimal $E^{*}$ of (3.1) satisfies $\lambda_{\min }\left(E^{*}\right) \geqslant$ $\frac{1}{d+1} n^{-d}$. 
Proof We already observed that $\eta_{2 d}(\mathcal{C})$ is independent of $n$. Now we estimate $\lambda_{\min }\left(E^{*}\right)$. For $s_{d}(t)=\frac{1}{d+1} \sum_{k=1}^{d} \sum_{j=0}^{k-1} t^{j}$, it holds that

$$
s_{d}(t)(1-t)=1-\frac{1}{d+1}\left(1+t+\cdots+t^{d}\right) .
$$

In (4.1), replacing $t$ by $\frac{1}{n}\|x\|_{2}^{2}$, we get that

$$
\frac{1}{n} s_{d}\left(\frac{1}{n}\|x\|_{2}^{2}\right)\left(\sum_{i=1}^{n}\left(1-x_{i}^{2}\right)\right)=1-\frac{1}{d+1}\left(1+\frac{1}{n}\|x\|_{2}^{2}+\cdots+\frac{1}{n^{d}}\|x\|_{2}^{2 d}\right) .
$$

Hence, there exists a symmetric matrix $E$ such that

$$
\frac{1}{d+1}\left(1+\frac{1}{n}\|x\|_{2}^{2}+\cdots+\frac{1}{n^{d}}\|x\|_{2}^{2 d}\right)=[x]_{d}^{T} E[x]_{d}, \quad \lambda_{\min }(E) \geqslant \frac{1}{d+1} n^{-d} .
$$

The polynomial $\frac{1}{n} s_{d}\left(\frac{1}{n}\|x\|_{2}^{2}\right)$ is SOS. So, Assumption 3.1 holds, and the optimal $E^{*}$ in (3.1) satisfies $\lambda_{\min }\left(E^{*}\right) \geqslant \frac{1}{d+1} n^{-d}$.

Clearly, Theorem 3.4(ii) and Lemma 4.3 imply the following corollary.

Corollary 4.4 Let $f \in \mathbb{R}[x]_{2 d}$, and $f_{\min }$ (resp., $f_{\max }$ ) be its minimum (resp., maximum) on the hypercube $\mathcal{C}=[-1,1]^{n}$. If $f_{\text {sos }}$ is the optimal value of $(1.2)$, then

$$
1 \leqslant \frac{f_{\max }-f_{\text {sos }}}{f_{\max }-f_{\min }} \leqslant \frac{(d+1) n^{d}}{\eta_{2 d}(\mathcal{C})} \sqrt{\left(\begin{array}{c}
n \\
2 d
\end{array}\right)} .
$$

So, $f_{\mathrm{sos}}$ is an $\mathcal{O}\left(n^{2 d}\right)$-approximation of $f_{\min }$ as $n \rightarrow \infty$.

4.3 Optimizing Square-Free Polynomials over a Hypercube

Consider the case that $S$ is the hypercube $\mathcal{C}=[-1,1]^{n}$ and $f(x)$ is square free, that is,

$$
f(x)=\sum_{\gamma \in \operatorname{Sfr}\left(\mathbb{N}_{2 d}^{n}\right)} f_{\gamma} x^{\gamma}, \quad \text { where } \operatorname{Sfr}\left(\mathbb{N}_{2 d}^{n}\right)=\mathbb{N}_{2 d}^{n} \cap\{0,1\}^{n} .
$$

Recall that $\operatorname{Sfr}[x]_{k}$ is the space of square free polynomials of degrees at most $k$.

\section{Lemma 4.5 It holds that}

$$
\chi\left(\operatorname{Sfr}[x]_{k}, \mathcal{C}\right) \leqslant \sqrt{3}^{k}, \quad \lambda \min \left(E^{*}\right) \geqslant \frac{1}{d+1} n^{-d} .
$$

Proof Let $p \in \operatorname{Sfr}[x]_{k}$ be such that $|p(x)| \leqslant 1$ for all $x \in \mathcal{C}$. If we write $p(x)=$ $\sum_{\gamma \in \operatorname{Sfr}\left(\mathbb{N}_{k}^{n}\right)} p_{\gamma} x^{\gamma}$, then a simple integration shows

$$
1 \geqslant \frac{1}{2^{n}} \int_{\mathcal{C}} p(x)^{2} d x=\sum_{\gamma \in \operatorname{Sfr}\left(\mathbb{N}_{k}^{n}\right)} p_{\gamma}^{2} \frac{1}{2^{n}} \int_{\mathcal{C}} x^{2 \gamma} d x=\sum_{\gamma \in \operatorname{Sfr}\left(\mathbb{N}_{k}^{n}\right)} p_{\gamma}^{2} 3^{-|\gamma|} \geqslant 3^{-k}\|p\|_{2}^{2} .
$$


Therefore, we have $\|p\|_{G} \leqslant\|p(x)\|_{2} \leqslant \sqrt{3}^{k}$. By definition (3.2), the first inequality follows immediately. The second one is a consequence of Lemma 4.3.

Lemma 4.5 implies $\chi\left(\operatorname{Sfr}[x]_{2 d}, \mathcal{C}\right) \leqslant 3^{d}$. Hence, Theorem 3.3 implies the following.

Corollary 4.6 Let $f \in \operatorname{Sfr}[x]_{2 d}$, and $f_{\min }$ (resp., $f_{\max }$ ) be its minimum (resp., maximum) on $[-1,1]^{n}$. If $f_{\text {sos }}$ is the optimal value of $(1.2)$, then it holds that

$$
f_{\max }-f_{\mathrm{sos}} \leqslant(d+1) \cdot(3 n)^{d}\left(f_{\max }-f_{\min }\right) .
$$

\subsection{Optimizing Polynomials over Boolean Sets}

Consider the case that $S$ is the boolean set $\{ \pm 1\}^{n}$. Then $m=2 n$ and

$$
g_{i}(x)=1-x_{i}^{2} \geqslant 0, \quad g_{n+i}(x)=-1+x_{i}^{2} \geqslant 0, \quad i=1, \cdots, n .
$$

The approximation bound of (1.2) for this case is given as below.

Corollary 4.7 Let $f \in \mathbb{R}[x]_{2 d}$, and $f_{\min }$ (resp., $f_{\max }$ ) be its minimum (resp., maximum) on $\{ \pm 1\}^{n}$. If $f_{\text {sos }}$ is the optimal value of (1.2), then it holds that

$$
f_{\max }-f_{\mathrm{sos}} \leqslant(d+1) n^{d}\left(f_{\max }-f_{\min }\right) .
$$

Proof From the proof of Lemma 4.3 we can find an SOS polynomial $s(x)$ satisfying

$$
s(x)\left(g_{1}(x)+\cdots+g_{n}(x)\right)=1-[x]_{d}^{T} E[x]_{d}, \quad \lambda_{\min }(E) \geqslant \frac{1}{d+1} n^{-d} .
$$

So the optimal $E^{*}$ in (3.1) for the set $\{ \pm 1\}^{n}$ must satisfy $\lambda_{\min }\left(E^{*}\right) \geqslant \frac{1}{d+1} n^{-d}$.

First, assume that $f \in \operatorname{Sfr}[x]_{2 d}$ is square free. We claim that

$$
\chi\left(\operatorname{Sfr}[x]_{2 d},\{ \pm 1\}^{n}\right) \leqslant 1 .
$$

To see this, suppose $p \in \operatorname{Sfr}[x]_{2 d}$ and $|p(x)| \leqslant 1$ for all $x \in\{ \pm 1\}^{n}$. If we write $p(x)=\sum_{\gamma \in \operatorname{Sfr}\left(\mathbb{N}_{2 d}^{n}\right)} p_{\gamma} x^{\gamma}$, then

$$
1 \geqslant \frac{1}{2^{n}} \sum_{u \in\{ \pm 1\}^{n}} p(u)^{2}=\sum_{\gamma \in \operatorname{Sfr}\left(\mathbb{N}_{2 d}^{n}\right)} p_{\gamma}^{2} \cdot \frac{1}{2^{n}} \sum_{u \in\{ \pm 1\}^{n}} u^{2 \gamma}=\sum_{\gamma \in \operatorname{Sfr}\left(\mathbb{N}_{2 d}^{n}\right)} p_{\gamma}^{2}=\|p\|_{2}^{2} .
$$

Hence, $\|p\|_{G} \leqslant\|p\|_{2} \leqslant 1$, and (4.2) is true by definition (3.2). So Theorem $3.3 \mathrm{im}$ plies the corollary when $f$ is square free.

Second, if $f$ is not square free, there exists $\hat{f} \in \operatorname{Sfr}[x]_{2 d}$ such that

$$
f(x)=\hat{f}(x) \quad \forall x \in\{ \pm 1\}^{n} .
$$

By the previous argument, the corollary is also true.

After a linear coordinate transformation, the approximation bound in Corollary 4.7 is also true for (1.2) when $S=\{0,1\}^{n}$. So we get the following: 
Corollary 4.8 Let $f \in \mathbb{R}[x]_{2 d}$, and $f_{\min }$ (resp., $f_{\max }$ ) be its minimum (resp., maximum) on $\{0,1\}^{n}$. If $f_{\text {sos }}$ is the optimal value of (1.2), then

$$
f_{\max }-f_{\text {sos }} \leqslant(d+1) n^{d}\left(f_{\max }-f_{\min }\right) .
$$

\subsection{Optimizing Polynomials over Quadratically Constrained Sets}

Consider the case that every $g_{i}(x)=[x]_{1}^{T} Q_{i}[x]_{1}$ is quadratic. If Assumption 3.1 holds, Theorem 3.4 can be applied to get a bound. The optimal value $\lambda_{\min }\left(E^{*}\right)$ of (3.1) can be estimated by using $Q_{i}$. If we set $d=1$, (3.1) reduces to

$$
\begin{cases}\max _{\lambda=\left(\lambda_{1}, \cdots, \lambda_{m}\right), A} & \lambda_{\min }(A) \\ \text { s.t. } & {[x]_{1}^{T}\left(A+\lambda_{1} Q_{1}+\cdots+\lambda_{m} Q_{m}\right)[x]_{1}=1,} \\ & \lambda_{1}, \cdots, \lambda_{m} \geqslant 0 .\end{cases}
$$

Let $\left(\lambda^{*}, A^{*}\right)$ be an optimal solution of (4.3).

Lemma 4.9 Suppose that every $g_{i}(x)$ is quadratic and $\lambda_{\min }\left(A^{*}\right)>0$ in (4.3). Then Assumption 3.1 holds, and $\lambda_{\min }\left(E^{*}\right) \geqslant \frac{1}{d}\left(\lambda_{\min }\left(A^{*}\right)\right)^{d}$ for $(3.1)$.

Proof Let $s(t)=\frac{1}{d} \sum_{k=1}^{d} \sum_{j=0}^{k-1} t^{j}$; then $s(t)(1-t)=1-\frac{1}{d}\left(t+\cdots+t^{d}\right)$. Plugging $t$ by $[x]_{1}^{T} A^{*}[x]_{1}$, we get the identity

$$
s\left([x]_{1}^{T} A^{*}[x]_{1}\right)\left(1-[x]_{1}^{T} A^{*}[x]_{1}\right)=1-\frac{1}{d}\left([x]_{1}^{T} A^{*}[x]_{1}+\cdots+\left([x]_{1}^{T} A^{*}[x]_{1}\right)^{d}\right) .
$$

From (4.3) we know that $1 \geqslant[x]_{1} A^{*}[x]_{1} \geqslant \lambda_{\min }\left(A^{*}\right)\left(1+\|x\|_{2}^{2}\right)$ for all $x \in S$. So there exists a symmetric matrix $E$ satisfying

$$
\frac{1}{d}\left([x]_{1}^{T} A^{*}[x]_{1}+\cdots+\left([x]_{1}^{T} A^{*}[x]_{1}\right)^{d}\right)=[x]_{d}^{T} E[x]_{d}, \quad \lambda_{\min }(E) \geqslant \frac{1}{d}\left(\lambda_{\min }\left(A^{*}\right)\right)^{d} .
$$

Let $\sigma_{i}(x)=\lambda_{i}^{*} s\left([x]_{1}^{T} A^{*}[x]_{1}\right)$ for every $i$, which are all SOS. Then we get

$$
\sigma_{1} g_{1}+\cdots+\sigma_{m} g_{m}=1-[x]_{d}^{T} E[x]_{d} .
$$

Hence, Assumption 3.1 holds, and the optimal value of (3.1) is at least $\frac{1}{d} \times$ $\left(\lambda_{\min }\left(A^{*}\right)\right)^{d}$.

Clearly, Theorem 3.4 and Lemma 4.9 imply the following corollary.

Corollary 4.10 Assume that every $g_{i}$ is quadratic and an optimal $A^{*}$ in (4.3) is positive definite. Let $f \in \mathbb{R}[x]_{2 d}$, and $f_{\min }$ (resp., $f_{\max }$ ) be the minimum (resp., maximum) of $f(x)$ on $S$. If $f_{\text {sos }}$ is the optimal value of (1.2), then it holds that

$$
1 \leqslant \frac{f_{\max }-f_{\text {sos }}}{f_{\max }-f_{\min }} \leqslant \min \left\{\frac{1}{\kappa_{2 d}(S)}, \frac{1}{\eta_{2 d}(S)} \sqrt{\left(\begin{array}{c}
n \\
2 d
\end{array}\right)}\right\} \frac{d}{\left(\lambda_{\min }\left(A^{*}\right)\right)^{d}} .
$$


Remark 4.11 If every $g_{i}(x)=[x]_{1}^{T} Q_{i}[x]_{1}$ is concave, we can get $\lambda_{\min }\left(A^{*}\right)$ exactly. Let $R=\max _{x \in S}\|x\|_{2}$ be the radius of $S$. Then $1 \geqslant[x]_{1}^{T} A^{*}[x]_{1} \geqslant \lambda_{\min }\left(A^{*}\right)(1+$ $\|x\|_{2}^{2}$ ) for all $x \in S$, and hence $\lambda_{\min }\left(A^{*}\right) \leqslant\left(1+R^{2}\right)^{-1}$. The quadratic function $1-\left(1+R^{2}\right)^{-1}[x]_{1}^{T}[x]_{1}$ is concave and nonnegative on $S$. If $\operatorname{int}(S) \neq \emptyset$, there exist $\left(\hat{\lambda}_{1}, \cdots, \hat{\lambda}_{m}\right) \geqslant 0$ satisfying

$$
1-\left(1+R^{2}\right)^{-1}\left(1+\|x\|_{2}^{2}\right)=\hat{\lambda}_{1}[x]_{1}^{T} Q_{1}[x]_{1}+\cdots+\hat{\lambda}_{m}[x]_{1}^{T} Q_{m}[x]_{1} .
$$

Thus, $\left(\hat{\lambda}_{1}, \cdots, \hat{\lambda}_{m}, \frac{1}{1+R^{2}} I_{n+1}\right)$ is feasible for (4.3), and $\lambda_{\min }\left(A^{*}\right) \geqslant\left(1+R^{2}\right)^{-1}$. So

$$
\lambda_{\min }\left(A^{*}\right)=\left(1+R^{2}\right)^{-1} .
$$

If some $g_{i}(x)=[x]_{1}^{T} Q_{i}[x]_{1}$ is not concave, it would be quite difficult to estimate $\lambda_{\min }\left(A^{*}\right)$ in (4.3) because (4.4) might not hold. However, if $R$ is known in advance, the redundant constraint $1-\left(1+R^{2}\right)^{-1}[x]_{1}^{T}[x]_{1} \geqslant 0$ could be added, and we still have $\lambda_{\min }\left(A^{*}\right)=\left(1+R^{2}\right)^{-1}$.

Example 4.12 (Multiunit ball) Suppose $x=\left(x^{(1)}, \cdots, x^{(m)}\right)$ where each $x^{(i)}$ is an $n_{i}$-dimensional vector, $g_{i}(x)=1-\left\|x^{(i)}\right\|_{2}^{2}, n_{i} \geqslant 2 d$, and $n_{1}+\cdots+n_{m}=n$. Such a set $S$ is called a multiunit ball. Observe that

$$
\frac{1}{m+1}\left(g_{1}+\cdots+g_{m}\right)=\frac{1}{m+1}\left(m-\|x\|_{2}^{2}\right)=1-[x]_{1}^{T} \frac{I_{n+1}}{m+1}[x]_{1} .
$$

So the optimal $A^{*}$ of (4.3) satisfies $\lambda_{\min }\left(A^{*}\right) \geqslant \frac{1}{m+1}$. Obviously, $\eta_{2 d}(S)>0$ depends only on $d$. Corollary 4.10 implies that an approximation bound $\mathcal{O}\left((m n)^{d}\right)$ holds for (1.2).

\section{References}

[1] Barvinok, A.: Estimating $L^{\infty}$ norms by $L^{2 k}$ norms for functions on orbits. Found. Comput. Math. 2(4), 393-412 (2002)

[2] Lasserre, J.: Global optimization with polynomials and the problem of moments. SIAM J. Optim. 11(3), 796-817 (2001)

[3] Lasserre, J.B.: Moments, Positive Polynomials and Their Applications. Imperial College Press, London (2009)

[4] Nie, J., Demmel, J., Sturmfels, B.: Minimizing polynomials via sum of squares over the gradient ideal. Math. Program., Ser. A 106(3), 587-606 (2006)

[5] Nie, J., Schweighofer, M.: On the complexity of Putinar's Positivstellensatz. J. Complex. 23, 135150 (2007)

[6] Pardalos, P.M., Vavasis, S.A.: Quadratic programming with one negative eigenvalue is NP-hard. J. Glob. Optim. 1(1), 15-22 (1991)

[7] Parrilo, P.: Semidefinite programming relaxations for semialgebraic problems. Math. Program., Ser. B 96(2), 293-320 (2003)

[8] Parrilo, P., Sturmfels, B.: Minimizing polynomial functions. In: Basu, S., Gonzalez-Vega, L. (eds.) Proceedings of the DIMACS Workshop on Algorithmic and Quantitative Aspects of Real Algebraic Geometry in Mathematics and Computer Science, March 2001, pp. 83-100. American Mathematical Society, Providence (2003) 
[9] Putinar, M.: Positive polynomials on compact semi-algebraic sets. Indiana Univ. Math. J. 42, 203$206(1993)$

[10] Reznick, B.: Some concrete aspects of Hilbert's 17th problem. In: Contemporary Mathematics, vol. 253, pp. 251-272. American Mathematical Society, Providence (2000)

[11] Wolkowicz, H., Saigal, R., Vandenberghe, L. (eds.): Handbook of Semidefinite Programming: Theory, Algorithms, and Applications. International Series in Operations Research \& Management Science, vol. 27. Kluwer Academic, Boston (2000) 